\title{
TRIANGULAR RESOLUTIONS AND EFFECTIVENESS FOR HOLOMORPHIC SUBELLIPTIC MULTIPLIERS
}

\author{
SUNG-YEON KIM AND DMITRI ZAITSEV
}

\begin{abstract}
A solution to the effectiveness problem in Kohn's algorithm for generating subelliptic multipliers is provided for domains that include those given by sums of squares of holomorphic functions (also including infinite sums). These domains are of particular interest due to their relation with complex and algebraic geometry and in particular seem to include all previously known cases. Furthermore, combined with a recent result of M. Fassina [Fa20, our effectiveness method allows to establish effective subelliptic estimates for more general classes of domains.

Our main new tool, a triangular resolution, is the construction of subelliptic multipliers decomposable as $Q \circ \Gamma$, where $\Gamma$ is constructed from pre-multipliers and $Q$ is part of a triangular system. The effectiveness is proved via a sequence of newly proposed procedures, called here metaprocedures, built on top of the Kohn's procedures, where the order of subellipticity can be effectively tracked. Important sources of inspiration are algebraic geometric techniques by Y.-T. Siu [S10, S17] and procedures for triangular systems by D.W. Catlin and J.P. D'Angelo [D95, CD10].

The proposed procedures are purely algebraic and as such can also be of interest for geometric and computational problems involving Jacobian determinants, such as resolving singularities of holomorphic maps.
\end{abstract}

\section{INTRODUCTION}

The Kohn's technique of subelliptic multipliers [Ko79] is one of the few known general techniques connecting a priori estimates for systems of partial differential equations (subelliptic estimates for the $\bar{\partial}$-Neumann problem [Ko79, Definition 1.11]) with tools from commutative algebra and algebraic geometry. Kohn's key innovations include refining subelliptic estimates by introducing multiplier functions [S17, §2.8], proposing purely algebraic procedures to generate new multipliers, and developing an algorithm to apply these procedures to obtain subelliptic estimates for realanalytic pseudoconvex domains of finite D'Angelo type. The reader is referred to [D95, DK99, S01, S02, Ko04, S05, S07, Ch06, S09, CD10, S10, S17] for more extensive details on subelliptic multipliers and Siu's accounts [S07, S09, S17] on their broad role and relation with other multipliers arising in complex and algebraic geometry.

On the other hand, the question of effectiveness, i.e. control of the Sobolev exponent of multipliers in Kohn's algorithm has remained an open problem since Kohn's work of 1979 (apart from the complex dimension 2, see [Ko79, §8], where it is based on fundamental results by Hörmander [Ho65] and Rothschild-Stein [RS76]). In higher dimension, the situation is much less understood,

2010 Mathematics Subject Classification. 32T25, 32T27, 32W05, 32S05, 32S10, 32S45, 32B10, 32V15, 32V35, $32 \mathrm{~V} 40$. 
in fact, examples of [He08] ( $§ 1.1$ in the preprint version) and [CD10, Proposition 4.4] in dimension 3 illustrate a lack of such control, see also [S17, §4.1] for a detailed explanation of this important phenomenon.

To tackle the effectiveness, Siu [S10, S17] introduced algebraic geometric techniques to obtain the effectiveness in the important case of special domains of finite type in dimension 3, with further indications how to proceed in the more general cases of special domains in higher dimension, and outlining a program to treat the more general real-analytic and smooth cases. A different effective procedure in Kohn's algorithm was given by D'Angelo [D95] and Catlin-D'Angelo [CD10, Section 5] for special domains given by so-called triangular systems of holomorphic functions. In N14 A.C. Nicoara proposed a construction for the termination of the Kohn algorithm in the real-analytic case with an indication of the ingredients needed for the effectivity. More recently, the authors of this article established another effective procedure in dimension 3 by means of a new tool of jet-vanishing orders [KZ18], where the reader is referred for further discussion and references.

After receiving a preprint of our paper, Y.-T. Siu told us about his unpublished proof of the effective termination of Kohns algorithm for special domains of arbitrary dimension by using descending induction on the dimension of the subvariety defined by effectively constructed multipliers and the techniques of multiplicities of Jacobian determinants and fiberwise differentiation without the complication of considering generalizations of the result of Skoda-Briançon.

1.1. Main results. In this paper we establish, in particular, the effectiveness for special domain of arbitrary dimension by means of the proposed new geometric tools of triangular resolutions and effective meta-procedures that are largely inspired by the algebraic geometric techniques by Y.-T. Siu [S10, S17] and procedures for triangular systems by D.W. Catlin and J.P. D'Angelo [D95, CD10.

Recall first the definition of special domains [Ko79, §7], [S17, §2.8]:

Definition 1.1. A special domain in $\mathbb{C}^{n+1}$ is one defined locally near each boundary point by

$$
\operatorname{Re}\left(z_{n+1}\right)+\sum_{j=1}^{N}\left|F_{j}\left(z_{1}, \ldots, z_{n}\right)\right|^{2}<0
$$

where $F_{1}, \ldots, F_{N}$ are holomorphic functions.

Next recall [Ko79, §7] that for special domains, Kohn's multiplier generation procedures can be formulated purely in terms of holomorphic functions, starting from the given set $S=\left\{F_{1}, \ldots, F_{N}\right\}$ of the functions in (1.1). We also adopt Siu's terminology [S10, S17], calling functions in $S$ premultipliers and the Sobolev exponent used in the modified subelliptic estimate with factor $f$, the order of subellipticity or just the order of the multiplier $f$.

Definition 1.2. For an initial set $S$ of pre-multipliers, the Kohn's procedures consist of: 
(P1) for $0<\varepsilon \leq 1 / 2$ and $f_{1}, \ldots, f_{n}$ either in $S$ or multipliers of order $\geq \varepsilon$, it follows that the Jacobian determinant

$$
g:=\operatorname{det}\left(\frac{\partial f_{i}}{\partial z_{j}}\right)=\frac{\partial\left(f_{1}, \ldots, f_{n}\right)}{\partial\left(z_{1}, \ldots, z_{n}\right)}
$$

is a multiplier of order $\geq \varepsilon / 2$;

(P2) for $0<\varepsilon<1, k, r \in \mathbb{N}_{\geq 1}, f_{1}, \ldots, f_{k}$ multipliers or order $\geq \varepsilon$, and $g$ a holomorphic function (germ) with $g^{r} \in\left(f_{1}, \ldots, f_{k}\right)$, it follows that $g$ is a multiplier of order $\geq \varepsilon / r$.

Note that it is (P2) that is responsible for the lack of effectiveness, as it is a priori not clear what root order $r$ needs to be used. To formulate our first result recall from D'Angelo [D79, D82, D93] and Siu [S10, S17] that the order of finite type can be estimated on both sides in terms of other quantities (see also Boas-Straube [BS92, Baouendi-Huang-Rothschild [BHR96], Fu-IsaevKrantz [FIK96], Fornaess-Lee-Zhang [FLZ14], Brinzanescu-Nicoara [BN15, BN19], McNeal-Mernik [MM17, D'Angelo [D17], Fassina [Fa19, Fa20], Huang-Yin [HY19], and the second author [Z19] for relations with other invariants). In this paper we use the multiplicity:

Definition 1.3. The multiplicity of an ideal $I \subset \mathcal{O}_{n, p}$ in the ring of germs at $p$ of holomorphic functions in $\mathbb{C}^{n}$ is the dimension of the quotient $\operatorname{dim}\left(\mathcal{O}_{n, p} / I\right) \leq \infty$. The multiplicity of a subset $S \subset \mathcal{O}_{n, p}$ is the multiplicity of its generated ideal. The multiplicity of a domain (1.1) at a boundary point $p$ is the multiplicity of the set of all germs at $p$ of the functions $F_{j}-F_{j}(p), 1 \leq j \leq N$.

To emphasize its algebraic nature, we formulate our first result purely in terms of the Kohn's procedures (P1) and (P2):

Theorem 1.4. For every initial subset $S \subset \mathcal{O}_{n, p}$ of finite multiplicity $\leq \nu$, there exists an effectively computable sequence of germs at $p$ of holomorphic functions $f_{1}, \ldots, f_{m}$, where $f_{m}=1$ and each $f_{j}$ is either in $S$ or is obtained by applying to $\left(f_{1}, \ldots, f_{j-1}\right)$ one of the Kohn's procedures (P1) or (P2). Furthermore, the number of steps $m$ and the root orders in (P2) are effectively bounded by functions depending only on $(n, \nu)$.

In the next result we apply Theorem 1.4 to obtain effective subelliptic estimates, with additional explicit bound for the order of subellipticity:

Theorem 1.5. There exists a positive function $\varepsilon: \mathbb{N}_{>0} \times \mathbb{N}_{>0} \rightarrow \mathbb{R}_{>0}$ such that for any domain (1.1) of finite multiplicity $\leq \nu$ at a boundary point $p$, a subelliptic estimate holds at $p$ with effectively bounded order of subellipticity $\geq \varepsilon(n, \nu)$. In fact, one can take

$$
\varepsilon(n, \nu)=\left(4(2 n+2)^{2(n \nu)^{(3 n)^{n+1}}}\right)^{-1} .
$$

Remark 1.6. Since the multiplicity $\nu$ of $I$ satisfies $\nu \leq t^{n}$ where $t$ is the type by a result of D'Angelo [D82, Theorem 2.7], an effective bound in terms of the type can be obtained by substituting $t^{n}$ for $\nu$ in (1.2). 
1.2. More general domains and applications. Due to the algebraic nature of Theorem 1.4 referring only to Kohn's procedures rather than to domains, the conclusion can be applied for more general domains, in conjunction with other effective procedures to obtain the initial set $S$ in Definition 1.2. Consider general domains given locally near a boundary point $p$ by

$$
\rho(z, \bar{z})<0,
$$

where $d \rho \neq 0$. Let $S \subset \mathcal{O}_{n, p}$ be the set of holomorphic function germs $F$ such that

$$
\left|\sum_{j} \frac{\partial F}{\partial z_{j}} v_{j}\right|^{2} \leq c\left(\sum_{j, k} \frac{\partial^{2} \rho}{\partial z_{j} \partial \bar{z}_{k}} v_{j} \bar{v}_{k}+\left|\sum_{j} \frac{\partial \rho}{\partial z_{j}} v_{j}\right|^{2}\right)
$$

for some $c>0$ and all $v_{1}, \ldots, v_{n}$. It follows from Fassina [Fa20, Remark 4.5] that $S$ consists of premultipliers in the sense of Siu [S17, §2.9] that can be used in Kohn's procedures. (In particular, if $\rho=\operatorname{Re} z_{n+1}+\phi\left(z_{1}, \ldots, z_{n}, \bar{z}_{1}, \ldots, \bar{z}_{n}\right)$ where $\phi$ is an infinite sum of squares of holomorphic functions $F_{j}$, each of $F_{j}$ is contained in $S$.) Hence we obtain the following application of Theorem 1.4;

Corollary 1.7. With $\varepsilon(n, \nu)$ given by (1.2), for a smooth pseudoconvex domain (1.3), assume that the multiplicity of $S$ is $\leq \nu$. Then a subelliptic estimate holds at $p$ with order $\geq \varepsilon(n, \nu)$. In particular, an effective subelliptic estimate holds for domains (1.2) of finite type with $\phi$ an infinite sum of squares of holomorphic functions.

We conclude this subsection by mentioning applications of (1) effective subelliptic estimates to local regularity with effective gain [KoN65], effective lower bound on the Bergman metric [M92] and control in the construction of peak functions [FM94] and (2) effective Kohn's algorithm (such as given by Theorem 1.4) to the construction of "bumping" functions, Kobayashi metric estimates and Hölder regularity of proper holomorphic maps [DF79].

1.3. Triangular resolutions and effective meta-procedures. In this section we introduce our main tools for establishing effectiveness. Recall that the crucial lack of effectiveness in (P2) (see Definition 1.2) is due to the order of the generated multiplier depending on the root order that can happen to be arbitrarily large.

To quantify this phenomenon, we call a procedure effective if the order of the new multiplier can be effectively estimated in terms of a quantity associated to the data that we call a complexity. We don't seek complexities of individual multipliers but rather of their finite tuples and tuples of their ideals, or more precisely, their filtrations. That is, we define, for a holomorphic map germ given by a tuple of pre-multipliers, and a filtration of ideals of multipliers, the notion of triangular resolution as follows:

Definition 1.8. A triangular resolution of length $k \geq 1$ and multi-order $\left(\mu_{1}, \ldots, \mu_{k}\right) \in \mathbb{N}^{k}$ of a pair $(\Gamma, \mathcal{I})$, where $\Gamma:\left(\mathbb{C}^{n}, 0\right) \rightarrow\left(\mathbb{C}^{n}, 0\right)$ is a holomorphic map germ and $I_{1} \subset \ldots \subset I_{k} \subset \mathcal{O}_{n, 0}$ a filtration $\mathcal{I}$ of ideals, is a system of holomorphic function germs $\left(h_{1}, \ldots, h_{k}\right)$ satisfying

$$
h_{j}=h_{j}\left(w_{j}, \ldots, w_{n}\right), \quad h_{j} \circ \Gamma \in I_{j}, \quad \operatorname{ord}_{w_{j}} h_{j}=\mu_{j}, \quad 1 \leq j \leq k .
$$


Remark 1.9. Triangular resolutions can be given an equivalent coordinate-free description by means of pairs $(s, h)$, where $s=\left(s_{1}, \ldots, s_{k}\right)$ is a sequence of holomorphic submersions

$$
\left(\mathbb{C}^{n}, 0\right) \stackrel{s_{1}}{\rightarrow}\left(\mathbb{C}^{n-1}, 0\right) \stackrel{s_{2}}{\rightarrow} \ldots \stackrel{s_{k}}{\rightarrow}\left(\mathbb{C}^{n-k}, 0\right)
$$

and $h=\left(h_{1}, \ldots, h_{k}\right)$ a $k$-tuple of holomorphic function germs satisfying

$$
h_{j} \in \mathcal{O}_{\mathbb{C}^{m-j+1}, 0}, \quad h_{j} \circ s_{j-1} \circ \ldots \circ s_{1} \circ \Gamma \in I_{j}, \quad \operatorname{mult}\left(h_{j}, s_{j}\right)=\mu_{j}, \quad j=1, \ldots, k .
$$

It is the multi-order of a triangular resolution that plays the role of the complexity as mentioned above in our effective procedures, that we call here meta-procedures in order to emphasize that each of them is a constructed as a sequence of Kohn's original procedures (P1) and (P2).

Remark 1.10. Since the multi-order depends on the resolution, we can define invariant polytopes $P_{k}=P_{k}(\Gamma, \mathcal{I}) \subset \mathbb{R}^{k}$ as convex hulls of all multi-orders arising in this way, that can be used for a fine-grained control of the effectiveness. As the construction of triangular resolution involves projections (submersions) to decreasing sequence of subspaces in the target space, $P_{k}$ can be seen as dual to the Newton-Okounkov polytopes [O96], [LM09, KaK12], where instead increasing sequences of subvarieties are used to compute the vanishing orders.

Our proof of the results from previous section is based on the the following effective metaprocedures involving triangular resolutions:

Theorem 1.11. For $0 \leq k \leq n$, the following hold:

(MP1) (Selection of a partial Jacobian). For any

$f=\left(f_{1}, \ldots, f_{k}\right) \in\left(\mathcal{O}_{n, 0}\right)^{k}, \quad \psi=\left(\psi_{k+1}, \ldots, \psi_{n}\right) \in\left(\mathcal{O}_{n, 0}\right)^{n-k}, \quad \operatorname{mult}(f, \psi) \leq \mu<\infty$,

there exist linear changes of the coordinates $z \in \mathbb{C}^{n}$ and of the components of $\psi$ in $\mathbb{C}^{n-k}$ such that for the partial Jacobian determinant

$$
J:=\frac{\partial\left(\psi_{k+1}, \ldots, \psi_{n}\right)}{\partial\left(z_{k+1}, \ldots, z_{n}\right)}
$$

the multiplicity mult $\left(f, J, \psi_{k+2}, \ldots, \psi_{n}\right)$ is effectively bounded by a function depending only on $(n, \mu)$.

(MP2) (Selection of a triangular resolution). For any

$$
f=\left(f_{1}, \ldots, f_{k}\right) \in\left(\mathcal{O}_{n, 0}\right)^{k}, \quad \psi=\left(\psi_{1}, \ldots, \psi_{n}\right) \in\left(\mathcal{O}_{n, 0}\right)^{n}
$$

with

$$
\operatorname{mult}\left(f_{1}, \ldots, f_{j}, \psi_{j+1}, \ldots, \psi_{n}\right) \leq \mu<\infty, \quad 0 \leq j \leq n
$$

there exists a triangular resolution $h=\left(h_{1}, \ldots, h_{k}\right)$ of $(\psi, \mathcal{I})$, where $\mathcal{I}$ is the filtration

$$
\left(f_{1}\right) \subset\left(f_{1}, f_{2}\right) \ldots \subset\left(f_{1}, \ldots, f_{k}\right),
$$

such that orders ord $w_{j} h_{j}$ are effectively bounded by functions depending only on $(n, \mu)$. 
(MP3) (Jacobian extension in a triangular resolution). For any

$$
\Gamma=(\phi, \psi) \in\left(\mathcal{O}_{n, 0}\right)^{k} \times\left(\mathcal{O}_{n, 0}\right)^{n-k},
$$

and filtration $\mathcal{I}$ of ideals $I_{1} \subset \ldots \subset I_{k+1} \subset \mathcal{O}_{n, 0}$ satisfying

$$
I_{k+1} \subset I_{k}+(J) \text {, }
$$

where $J$ is the Jacobian determinant of $\Gamma$, let $h=\left(h_{1}, \ldots, h_{k+1}\right)$ be a triangular resolution with

$$
\operatorname{ord}_{z_{j}} h_{j} \leq \mu<\infty, \quad 1 \leq j \leq k
$$

Then $h_{k+1} \circ \Gamma$ can be obtained by holomorphic Kohn's procedures (P1) and (P2) starting with the initial set of components of $\psi$ and the ideal $I_{k}$, where the number of procedures and the root order in (PQ) are effectively bounded by a function depending only on $(n, \mu)$.

The proof for each of the statements in (MP1), (MP2) and (MP3) will be provided in their more precise versions with explicit estimates in Propositions 4.1, 5.1 and 6.1 respectively. All three meta-procedures are subsequently combined in Corollary 7.1 providing an iteration step in the main construction, where one more multiplier is added to the system. For reader's convenience, here is a self-contained variant of Corollary 7.1 .

Corollary 1.12. For any integer $\nu \geq 1$, initial system of pre-multipliers $\psi_{0}=\left(\psi_{0,1}, \ldots, \psi_{0, n}\right)$ of finite type $\leq \nu$, and $1 \leq k \leq n$, there exist:

(1) holomorphic coordinates $\left(z_{1}, \ldots, z_{n}\right)$ chosen among linear combinations of any given holomorphic coordinate system;

(2) systems of pre-multipliers $\psi_{k}=\left(\psi_{k, k+1}, \ldots, \psi_{k, n}\right)$ chosen among generic linear combination of the given ones, and associated maps

$$
\Gamma_{k}(z):=\left(z_{1}, \ldots, z_{k}, \psi_{k, k+1}(z), \ldots, \psi_{k, n}(z)\right) ;
$$

(3) systems of multipliers $f_{k}=\left(f_{k, 1}, \ldots, f_{k, k}\right)$ obtained via effective meta-procedures applied to $\left(\psi_{k-1}, f_{k-1}\right)$ (where $f_{0}$ is empty);

(4) decompositions of the form $f_{k, j}=Q_{k, j} \circ \Gamma_{k}, j=1, \ldots, k$, where each $Q_{k, j}=Q_{k, j}\left(w_{j}, \ldots, w_{n}\right)$ is a holomorphic function depending only on the last $n-j+1$ coordinates;

(5) positive functions $\varepsilon_{k, j}(n, \nu)>0$ such that the order of subellipticity of each $f_{k, j}$ is $\geq$ $\varepsilon_{k, j}(n, \nu)$.

Finally the estimates from Theorem 1.4 are provided by Corollary 7.2 .

Remark 1.13. Since all meta-procedures (MP1-3) in Theorem1.11 are purely algebraic, they can be applied to geometric problems beyond subelliptic estimates. For instance, consider a holomorphic map $\phi: V \rightarrow \mathbb{C}^{m}$ on a singular (not necessarily reduced) subvariety $V \subset \mathbb{C}^{n}$ given by a sheaf of ideals $\mathcal{I}$, and look for canonical ways of resolving the singularity of $\phi$. Here adding a Jacobian determinant in (P1) to the ideal sheaf $\mathcal{I}$ can be regarded as a step in simplifying the singularity of $\phi$, since in the regular case the Jacobian would be a unit. The singularity itself can then be encoded by trees whose edges correspond to (MP1-3) and that are labeled by multi-orders of the 
triangular resolutions. Such trees can be used to obtain more refined effective subelliptic estimates and put in a broader context of labeled trees encoding singularities, see e.g. [GGP19.

We conclude with Section 8 illustrating the use of our effective algorithm for a concrete class of examples with arbitrary high order perturbations.

Acknowledgement. We would like to thank Professors J.P. D'Angelo, X. Huang and Y.-T. Siu for their interest and helpful comments.

\section{Preliminaries}

2.1. Multiplicity and degree. Denote by $\mathcal{O}=\mathcal{O}_{n, p}$ the ring of germs at a point $p$ of holomorphic functions in $\mathbb{C}^{n}$. Since our considerations are for germs at a fixed point, we shall assume $p=0$ unless specified otherwise.

Recall that an ideal $\mathcal{I} \subset \mathcal{O}$ is of finite type if $\operatorname{dim} \mathcal{O} / \mathcal{I}<\infty$, or equivalently the (germ at 0 of the) zero variety $\mathcal{V}(\mathcal{I})$ is zero-dimensional at 0 . In the latter case, the classical algebraic intersection multiplicity of $\mathcal{I}$ (see e.g. [Fu84, $§ 1.6, \S 2.4]$ ) is defined as

$$
\text { mult } \mathcal{I}:=\operatorname{dim} \mathcal{O} / \mathcal{I}
$$

Similarly, for a germ of holomorphic map $\psi:\left(\mathbb{C}^{n}, 0\right) \rightarrow\left(\mathbb{C}^{n}, 0\right)$, we have mult $\psi:=$ mult $(\psi)$, where $(\psi)$ is the ideal generated by the components of $\psi$, and the quotient $\mathcal{O} /(\psi)$ is the local algebra of $\psi$ (see e.g. [AGV85]). More generally (cf. [D93, §2.4]), for every integer $0 \leq d<n$, define the d-multiplicity by

$$
\text { mult }{ }_{d} \mathcal{I}:=\min \operatorname{dim} \mathcal{O} /\left(\mathcal{I}+\left(L_{1}, \ldots, L_{d}\right)\right),
$$

where the minimum is taken over sets of $d$ linear functions $L_{j}$ on $\mathbb{C}^{n}$. The same minimum is achieved when $L_{j}$ are germs of holomorphic functions with linearly independent differentials, as can be easily shown by a change of coordinates linearlizing the functions. In particular, the $d$-multiplicity of an ideal is a biholomorphic invariant. In a similar vein, given a collection $\phi=\left(\phi_{1}, \ldots, \phi_{n-d}\right) \in \mathcal{O}_{n}$, of $n-d$ function germs, we write

$$
\operatorname{mult}(\phi)=\operatorname{mult}\left(\phi_{1}, \ldots, \phi_{n-d}\right):=\min \operatorname{dim} \mathcal{O} /\left(\phi_{1}, \ldots, \phi_{n-d}, L_{1}, \ldots, L_{d}\right),
$$

where $L_{j}$ are as above. That is, we will adopt the following convention:

Convention. For every $0 \leq k \leq n$ and a $k$-tuple of holomorphic function germs $\phi_{1}, \ldots, \phi_{k}$, their multiplicity mult $\left(\phi_{1}, \ldots, \phi_{k}\right)$ is always assumed to be the $(n-k)$-multiplicity, i.e. with $(n-k)$ generic linear functions added to the ideal.

Further recall that the degree $\operatorname{deg}(\psi)$ of a germ (also called "index" in [AGV85]) of a finite holomorphic map $\psi:\left(\mathbb{C}^{n}, 0\right) \rightarrow\left(\mathbb{C}^{n}, 0\right)$ is the minimum $m$ such that $\psi$ restricts to a ramified $m$-sheeted covering between neighborhoods of 0 in $\mathbb{C}^{n}$. Both integers are known to coincide (see e.g. [ELT77, AGV85, D93]):

Theorem 2.1 ([AGV85, §4.3]). Let $\psi:\left(\mathbb{C}^{n}, 0\right) \rightarrow\left(\mathbb{C}^{n}, 0\right)$ be germ of finite holomorphic map. Then

$$
\operatorname{mult}(\psi)=\operatorname{deg} \psi \text {. }
$$


2.2. Semi-continuity of multiplicity and application. The following lemma is straightforward consequence of the definition of multiplicity:

Lemma 2.2. Let $\psi_{t}:\left(\mathbb{C}^{m}, 0\right) \rightarrow\left(\mathbb{C}^{k}, 0\right)$ be a continuous family of germs of holomorphic maps, in the sense that all coefficients of the power series expansion of $\psi_{t}$ depend continuously on $t \in \mathbb{R}^{m}$. Then mult $\left(\psi_{t}\right)$ is upper semicontinuous in $t$.

In the following we keep using the notation (2.3).

Corollary 2.3. For every germs

$$
(f, g):\left(\mathbb{C}^{n+m}, 0\right) \rightarrow\left(\mathbb{C}^{n}, 0\right) \times\left(\mathbb{C}^{m}, 0\right),
$$

we have

$$
\text { mult } f \leq \operatorname{mult}(f, g) \text {. }
$$

Proof. Consider the family $g_{t}:=g+t\left(z_{1}, \ldots, z_{m}\right)$. Then Lemma 2.2 implies that

$$
\operatorname{mult}\left(f, g_{t}\right) \leq \operatorname{mult}(f, g)
$$

for $t$ near 0 . Choosing $t$ such that $g_{t}$ is immersive at 0 , we can change holomorphic coordinates in $\mathbb{C}^{n+m}$ to make $g_{t}$ linear. Then the desired conclusion follows from the definition of multiplicity.

2.3. Siu's lemma on effective mixed multiplicity. An important ingredient is the following consequence from Siu's lemma on selection of linear combinations of holomorphic functions for effective multiplicity [S10, (III.3)] combined with effective comparison of the invariants of holomorphic map germs [S10, (I.3-4)] (see also [D93, §2.2]):

Lemma 2.4 (Siu). Let $0 \leq j \leq q \leq n \leq N$, and $f_{1}, \ldots, f_{j}, F_{1}, \ldots, F_{N}$ be holomorphic function germs in $\mathcal{O}_{n, 0}$ such that

$$
\mu:=\operatorname{mult}\left(f_{1}, \ldots, f_{j}\right)<\infty, \quad \nu:=\operatorname{mult}\left(F_{1}, \ldots, F_{N}\right)<\infty .
$$

Then

$$
\operatorname{mult}\left(f_{1}, \ldots, f_{j}, G_{1}, \ldots, G_{n-q}\right) \leq \mu \nu^{n-q}
$$

holds for generic linear combinations $G_{1}, \ldots, G_{n-q}$ of $F_{j}$ 's.

\section{Effective Nullstellensatz}

We shall need the following effectiveness lemma that essentially follows the lines of Heier [He08], (see also [LJT08] for related techniques):

Lemma 3.1 (Effective Nullstellensatz). Let $\phi_{1}, \ldots, \phi_{k}, f \in \mathcal{O}_{n, 0}$ satisfy

$$
\mu:=\operatorname{mult}\left(\phi_{1}, \ldots, \phi_{k}\right)<\infty, \quad f \in \sqrt{\left(\phi_{1}, \ldots, \phi_{k}\right)} .
$$

Then

$$
f^{n \mu} \in\left(\phi_{1}, \ldots, \phi_{k}\right) \text {. }
$$


Proof. Let $(\phi):=\left(\phi_{1}, \ldots, \phi_{k}\right)$ be the ideal of $\mathcal{O}_{n}$ generated by $\phi_{1}, \ldots, \phi_{k}$. By the definition of multiplicity, for a generic choice of linear functions $L_{k+1}, \ldots, L_{n}$, one has

$$
\mu=\operatorname{mult}\left(\phi_{1}, \ldots, \phi_{k}\right)=\operatorname{mult}\left(\phi_{1}, \ldots, \phi_{k}, L_{k+1}, \ldots, L_{n}\right) .
$$

By the semicontinuity of multiplicity, there exists a neighborhood $U$ of 0 such that the multiplicity of $\left(\phi_{1}, \ldots, \phi_{k}\right)$ at $p \in U \cap\{\phi=0\}$ is less or equal to $\mu$. After shrinking $U$ if necessary, we may assume that there exist finite number of generators $h_{1}, \ldots, h_{m}$ of $\sqrt{\left(\phi_{1}, \ldots, \phi_{k}\right)}$ such that each $h_{j}$ is holomorphic on $U$.

Let $p \in U \cap\{\phi=0\}$. Choose $k$-dimensional generic linear subspace passing through $p$ defined by $L_{k+1}^{p}, \ldots, L_{n}^{p}$ such that

$$
\operatorname{mult}\left(\phi_{1}, \ldots, \phi_{k}, L_{k+1}^{p}, \ldots, L_{n}^{p}\right) \leq \mu \text {. }
$$

Choose generic linear combinations $\psi_{1}, \ldots, \psi_{k-1}$ of components of $\phi$ and define irreducible curves $C_{1}, \ldots, C_{\delta}$ by

$$
\bigcup_{j} C_{j}=\mathcal{V}\left(\psi_{1}, \ldots, \psi_{k-1}, L_{k+1}^{p}, \ldots, L_{n}^{p}\right) .
$$

Let $\gamma_{j}:(\Delta, 0) \rightarrow\left(C_{j}, p\right)$ be a local parametrization of $C_{j}$ at $p \in C_{j} \cap\{\phi=0\}$. Since

$$
h^{\mu} \in\left(\phi_{1}, \ldots, \phi_{k}, L_{k+1}^{p}, \ldots, L_{n}^{p}\right)
$$

for any $h \in \mathcal{O}_{n, p}$ with $h(p)=0$ (see e.g. [D93, §2.3.3], [S10, Lemma I.5]), we obtain for each $j=1, \ldots, \delta$, and $\ell=1, \ldots, m$,

$$
\mu \cdot \operatorname{ord}\left(h_{\ell} \circ \gamma_{j}\right)=\operatorname{ord}\left(h_{\ell}^{\mu} \circ \gamma_{j}\right) \geq \min _{\ell=1, \ldots, k} \operatorname{ord}\left(\phi_{\ell} \circ \gamma_{j}\right) \text {. }
$$

Hence

$$
\tau_{p}:=\max _{j} \frac{\operatorname{ord}\left(\gamma_{j}^{*}(\phi)\right)}{\operatorname{ord}\left(\gamma_{j}^{*}(\sqrt{(\phi)})\right)} \leq \mu,
$$

where $(\phi)$ denotes the generated ideal as before.

As in Heier [He08, define the type $\mathcal{T}(\phi)$ by

$$
\mathcal{T}(\phi):=\sup _{\gamma \in \Gamma_{0}}\left\{\frac{\operatorname{ord}\left(\gamma^{*}(\phi)\right)}{\operatorname{ord}\left(\gamma^{*}(\sqrt{(\phi)})\right)}\right\},
$$

where $\Gamma_{0}$ is the set of germs of local holomorphic curves $\gamma:(\Delta, 0) \rightarrow\left(\mathbb{C}^{n}, p\right)$ with $p$ in the zero set of $(\phi)$ whose image is not contained in $\mathcal{V}(\phi)$. We claim that for sufficiently small $U$,

$$
\sup _{p \in U} \tau_{p}=\mathcal{T}(\phi)
$$

for a generic choice of $\psi=\left(\psi_{1}, \ldots, \psi_{k-1}\right)$ and $L_{k+1}^{p}, \ldots, L_{n}^{p}$. Then the desired statement follows from [He08, Theorem 3.2].

To prove the claim (3.1), we follow [He08, Section 3.2] by letting

$$
B l_{\phi}(U) \rightarrow U
$$


be the blowing up of the ideal $(\phi)$ on an open neighborhood $U$ of $0 \in \mathbb{C}^{n}$ and let $X^{+} \rightarrow B l_{\phi}(U)$ be the normalization of the blowing up. Then there exists an effective Cartier divisor $F$ such that

$$
(\phi) \cdot \mathcal{O}_{X^{+}}=\mathcal{O}_{X^{+}}(-F),
$$

where $(\phi)$ is identified with its pullback. Write

$$
F=\sum_{i=1}^{s} r_{i} E_{i}
$$

After shrinking $U$ if necessary, we may assume that

$$
\bigcap_{i} \sigma\left(E_{i}\right) \ni 0
$$

where $\sigma: X^{+} \rightarrow U$ is the natural map given by blowing up and normalization. Let

$$
m_{i}:=\operatorname{ord}_{E_{i}}\left(h_{1} \circ \sigma, \ldots, h_{m} \circ \sigma\right), i=1, \ldots, s
$$

be the vanishing order of the ideal $\left(h_{1} \circ \sigma, \ldots, h_{m} \circ \sigma\right)$ at generic points of $E_{i}$. By Proposition 3.4 of [He08],

$$
\mathcal{T}(\phi)=\max _{i}\left\{\frac{r_{i}}{m_{i}}\right\}
$$

Then claim can be proved by following the arguments of the proof of Theorem 3.5 of [He08]. For reader's convenience, we include details as follows.

Assume that

$$
\frac{r_{1}}{m_{1}}=\max \left\{\frac{r_{i}}{m_{i}}\right\} .
$$

Choose a function $g$ whose divisor is $E_{1}$ :

$$
E_{1}=\operatorname{div}(g) .
$$

Then for generic $\tilde{p} \in E_{1}$ and a curve $\tilde{\gamma}$ whose image is not contained in $E_{1}$ and $\tilde{\gamma}(0)=\tilde{p}$, we obtain

$$
\frac{\operatorname{ord}\left(\tilde{\gamma}^{*}(\phi \circ \sigma)\right)}{\operatorname{ord}\left(\tilde{\gamma}^{*}(h \circ \sigma)\right)}=\frac{\operatorname{ord}\left(\tilde{\gamma}^{*}\left(g^{r_{1}}\right)\right)}{\operatorname{ord}\left(\tilde{\gamma}^{*}\left(g^{m_{1}}\right)\right)}=\frac{r_{1}}{m_{1}} .
$$

Let $p=\sigma(\tilde{p}) \in\{\phi=0\} \cap U$ and let $\gamma_{j}:(\mathbb{C}, 0) \rightarrow\left(C_{j}, p\right), j=1, \ldots, \delta$ be as before. Let

$$
D_{j}=\operatorname{div}\left(\psi_{j}\right), \sigma^{*}\left(D_{j}\right)=F+\widetilde{D}_{j}, \quad j=1, \ldots, k-1
$$

and let

$$
G_{j}=\operatorname{div}\left(L_{j}^{p}\right), \sigma^{*}\left(G_{j}\right)=\widetilde{G}_{j}, \quad j=k+1, \ldots, n .
$$

Then

$$
\widetilde{D}_{1} \cap \cdots \cap \widetilde{D}_{k-1} \cap \widetilde{G}_{k+1} \cap \cdots \cap \widetilde{G}_{n}
$$


is a union of curves. Since normalization $X^{+} \rightarrow B l_{\phi}(U)$ is a finite map, by Bertini's Theorem, for generic choice of $\tilde{p} \in E_{1}, \psi_{1}, \ldots, \psi_{k-1}$ and $L_{k+1}^{p}, \ldots, L_{n}^{p}$, one of the curves, say $\tilde{\gamma}_{1}$, the lift of $\gamma_{1}$, is a smooth curve meeting $E_{1}$ at some generic point $\tilde{\gamma}_{1}(0) \in \sigma^{-1}(p)$. Since $\gamma_{1}=\sigma \circ \tilde{\gamma}_{1}$, we obtain

$$
\frac{\operatorname{ord}\left(\gamma_{1}^{*}(\phi)\right)}{\operatorname{ord}\left(\gamma_{1}^{*}(h)\right)}=\frac{\operatorname{ord}\left(\tilde{\gamma}_{1}^{*}(\phi \circ \sigma)\right)}{\operatorname{ord}\left(\tilde{\gamma}_{1}^{*}(h \circ \sigma)\right)}=\frac{r_{1}}{m_{1}}
$$

achieving the maximum in (3.2) proving (3.1) as desired.

\section{Multiplicity estimates for JaCobian Determinants}

We have the following more precise version of the meta-procedure (MP1) in Theorem 1.11 that can be of independent interest:

Proposition 4.1 (Selection of a partial Jacobian determinant). Let

$$
(f, \psi):\left(\mathbb{C}^{m+d}, 0\right) \rightarrow\left(\mathbb{C}^{m} \times \mathbb{C}^{d}, 0\right), \quad m, d \geq 1,
$$

be a finite holomorphic map germ. Then after a linear change of holomorphic coordinates $\left(z_{1}, \ldots, z_{m+d}\right)$ around 0 , one has

$$
\text { mult }\left(f, \frac{\partial\left(\psi_{1}, \ldots, \psi_{d}\right)}{\partial\left(z_{1}, \ldots, z_{d}\right)}\right) \leq d \cdot \operatorname{mult} f \cdot \operatorname{mult}(f, \psi) \text {. }
$$

Proof. Let

$$
V:=\{f=0\} \subset \mathbb{C}^{m+d}
$$

be the germ at 0 of a subvariety defined by $f$. By additivity of the intersection number, we may assume that $V$ is irreducible. By Theorem 2.0.2 of [W08, we can choose an embedded desingularization $r: \widetilde{M} \rightarrow U$, where $U$ is a neighborhood of 0 in $\mathbb{C}^{m+d}$, with exceptional divisor $E \subset \widetilde{M}$, such that the strict transform $\widetilde{V} \subset \widetilde{M}$ of $V$ is regular. We write $\sigma: \widetilde{V} \rightarrow V$ for the restriction of $r$. Since $V$ is of pure dimension $d, \widetilde{V}$ is a smooth variety of dimension $d$. Let

$$
E=\sum_{j} c_{j} E_{j}
$$

where $E_{j}$ are irreducible. Note that $\widetilde{V}$ has only simple normal crossings with $E$. Since the desingularization $r$ of [W08] is given by a sequence of blow-ups at smooth center which has simple normal crossings with exceptional divisors of the previous blow-up, we may assume $E_{j}$ is smooth. Note further that the blow-up centers of $\sigma$ are disjoint from the set $V_{\text {reg }}$ of points where $V$ is smooth.

Let

$$
\mu:=\operatorname{mult}(f, \psi)
$$

and

$$
\Psi:=\psi \circ \sigma: \widetilde{V} \rightarrow \mathbb{C}^{d} .
$$

Choose open neighborhoods $W_{j} \subset \widetilde{V}$ of $E_{j} \cap \widetilde{V}$. Then $\cup_{j} \sigma\left(W_{j}\right)$ is an open neighborhood of 0 in $V$ and therefore, $\cup_{j} \Psi\left(W_{j}\right)$ is an open neighborhood of $0 \in \mathbb{C}^{d}$. Since there are only finite number of divisors $E_{j}$, there exists $j_{0}$ such that $\Psi\left(W_{j_{0}}\right)$ contains an open sector attached to 0 . Since each 
blow-ups has exceptional divisors of the form $M \times C$, where $C$ is a smooth center and $M$ is a compact manifold, we can cover $E_{j_{0}}$ with finite number of open sets of the polydisc form and at least one of them maps to a set containing open sector attached to $0 \in \mathbb{C}^{d}$ via $\Psi$.

Consider $\Psi^{-1}(0)$. Then $\Psi^{-1}(0)$ is a subvariety in $\widetilde{V}$ of codimension $k \leq d$. Choose a regular point $p_{0} \in \Psi^{-1}(0)$ and a local coordinate system $(x, y)=\left(x_{1}, \ldots, x_{k}, y_{k+1}, \ldots, y_{d}\right)$ centered at $p_{0}=(0, \ldots, 0)$ such that

$$
\Psi^{-1}(0)=\left\{x_{1}=\cdots=x_{k}=0\right\} .
$$

Assume that the image of a neighborhood $\left\{(x, y): x \in U_{1}, y \in U_{2}\right\}$ of $p_{0} \in \widetilde{V}$ via $\Psi$ contains an open sector attached to 0 . For $y \in U_{2}$, define

$$
N_{y}=\left\{(x, y): x \in U_{1}\right\} \subset \widetilde{V} .
$$

Then for generic $y$, the map $\sigma_{y}:=\sigma_{N_{y}}: N_{y} \rightarrow V$ is one to one and $\Psi_{y}:=\Psi_{N_{y}}: N_{y} \rightarrow \Psi\left(N_{y}\right)$ is a finite branched holomorphic covering of sheet number $\leq \mu$.

First we will show that

$$
\operatorname{dim} \mathcal{O}_{k} /\left(\Psi_{y}\right) \leq \mu,
$$

where $\left(\Psi_{y}\right)$ is an ideal generated by the components of $\Psi_{y}$. It is enough to show that

$$
\operatorname{dim} \mathcal{O}_{k} /\left(\Psi_{0}\right) \leq \mu .
$$

Choose a regular curve $\gamma:(\mathbb{C}, 0) \rightarrow\left(N_{0}, 0\right)$. Suppose

$$
\operatorname{dim} \mathcal{O}_{1} / \gamma^{*}\left(\Psi_{0}\right)>\mu
$$

Then the equation $\Psi_{0}(\gamma(\zeta))=\Psi_{0}(\gamma(a))$ has at least $\mu+1$ solutions for generic $a \in \mathbb{C}$ sufficiently close to 0 , which contradicts the assumption that $\Psi_{0}$ is at most $\mu$ to one. Therefore (4.2) holds. In particular,

which implies

$$
x_{i}^{\mu} \in\left(\Psi_{y}\right), \quad i=1, \ldots, k,
$$

$$
\left.\|x\|^{\mu} \leq c(y) \| \Psi_{y}(x)\right) \|, \quad(x, y) \in N_{y}
$$

for some function $c(y)$. By considering the expression of $x_{i}^{\mu}$ in terms of the components of $\Psi_{y}$, we can choose a point $y_{0}$ sufficiently close to 0 and a neighborhood $U_{2}^{\prime} \subset U_{2}$ of $y_{0}$ such that

$$
\left.\|x\|^{\mu} \leq c_{0} \| \Psi_{y}(x)\right) \|, \quad(x, y) \in N_{y}, \quad y \in U_{2}^{\prime}
$$

for some constant $c_{0}$.

Let $J(x, y)$ be the Jacobian determinant of $\Psi$. Since $J \equiv 0$ on $\Psi^{-1}(0), J$ is in the ideal generated by $x=\left(x_{1}, \ldots, x_{k}\right)$. Consider the power series expansion

$$
J(x, y)=\sum_{\alpha} c_{\alpha}(y) x^{\alpha}
$$

at $\left(0, y_{0}\right)$.

We will show:

Claim. There exists $\alpha$ with $\|\alpha\| \leq d \mu$ such that $c_{\alpha} \not \equiv 0$. 
Assuming the claim does not hold, after shrinking $U_{1}$ and $U_{2}^{\prime}$ if necessary, we obtain

$$
\mid J(x, y)) \leq c_{1}\|x\|^{T}, \quad(x, y) \in U_{1} \times U_{2}^{\prime}
$$

for some $T>d \mu$, where the constant $c_{1}$ is independent of $x$ and $y$. Choose a sector

$$
S \subset \Psi\left(U_{1} \times U_{2}^{\prime}\right) \backslash \Psi(\{J=0\} \cup E) .
$$

Then

$$
|J(x, y)| \leq c_{1}\|x\|^{T}, \quad x \in(\Psi)^{-1}(S) .
$$

Let $\omega$ and $\widetilde{\omega}$ be standard volume forms on $\mathbb{C}^{d}$ and $\widetilde{V}$ respectively. Then (4.6) implies

$$
\left(\Psi^{*} \omega\right)(x, y) \leq c_{2}\|x\|^{2 T} \cdot \widetilde{\omega}, \quad(x, y) \in(\Psi)^{-1}(S) .
$$

Write $B_{\varepsilon} \subset \mathbb{C}^{d}$ for the ball of radius $\varepsilon$ centered at 0 and consider the sectorial region

$$
D_{\varepsilon}:=S \cap\left(B_{2 \varepsilon} \backslash \overline{B_{\varepsilon}}\right) \subset \mathbb{C}^{d},
$$

whose volume satisfies

$$
\operatorname{volume}\left(D_{\varepsilon}\right)=\int_{D_{\varepsilon}} \omega \geq c_{3} \varepsilon^{2 d}
$$

Since the volume of $\delta$-ball in $U_{1}$ does not exceed the volume of the $\delta$-ball in $\mathbb{C}^{k}$ up to a constant, we have

$$
\int_{\Psi^{-1}\left(D_{\varepsilon}\right)} \Psi^{*} \omega \leq c_{4} \delta^{2 T} \int_{B_{\delta} \times U_{2}^{\prime}} \widetilde{\omega} \leq c_{5} \delta^{2 T+2 k},
$$

for all $\delta$ with $\Psi^{-1}\left(D_{\varepsilon}\right) \subset B_{\delta} \times U_{2}^{\prime}$. In view of (4.3), we can choose $\delta=c_{6} \varepsilon^{1 / \mu}$ in (4.8). Then, together with (4.7), we obtain

$$
\varepsilon^{2 d} \leq c_{8} \varepsilon^{(2 T+2 k) / \mu}
$$

for any sufficiently small $\varepsilon>0$, which is a contradiction proving the claim.

Since $J$ is holomorphic, there exist an integer $m \leq d \mu$ and a variety $X$ such that for each $p \in \Psi^{-1}(0) \backslash X, \Psi^{-1}(0)$ is smooth at $p$ and there exists a holomorphic coordinates $(x, y)=$ $\left(x_{1}, \ldots, x_{k}, y_{k+1}, \ldots, y_{d}\right)$ at $p$ such that

$$
\Psi^{-1}(0)=\{x=0\}
$$

and

$$
J(x, y)=x_{1}^{m}+\sum_{\ell<m} c_{\ell}\left(x^{\prime}, y\right) x_{1}^{\ell}
$$

where $x^{\prime}=\left(x_{2}, \ldots, x_{k}\right)$. Choose a point $p_{1} \in \Psi^{-1}(0) \backslash X$ and a generic smooth holomorphic curve $\gamma:(\mathbb{C}, 0) \rightarrow\left(\widetilde{V}, p_{1}\right)$ such that

By chain rule, we obtain

$$
\operatorname{ord}(J \circ \gamma)=m \text {. }
$$

$$
\sigma^{*}\left(\left.d \psi_{1} \wedge \cdots \wedge d \psi_{d}\right|_{V}\right)=J d w_{1} \wedge \cdots \wedge d w_{d}
$$


where $w=\left(w_{1}, \ldots, w_{d}\right)$ is a coordinate system of $\widetilde{V}$ centered at $p_{1}$. Therefore we obtain

$$
\sum_{\left(i_{1}, \ldots, i_{d}\right)} \frac{\partial\left(\psi_{1}, \ldots, \psi_{d}\right)}{\partial\left(z_{i_{1}}, \ldots, z_{i_{d}}\right)}(\sigma \circ \gamma(\zeta)) \frac{\partial\left(\Sigma_{i_{1}}, \ldots, \Sigma_{i_{d}}\right)}{\partial\left(w_{1}, \ldots, w_{d}\right)}(\gamma(\zeta))=J(\gamma(\zeta)),
$$

where $\sigma=\left(\Sigma_{1}, \ldots, \Sigma_{m+d}\right)$. Since $\sigma$ is one to one outside the exceptional divisor of $\sigma$, we can choose $\left(i_{1}, \ldots, i_{d}\right)$ such that

$$
\frac{\partial\left(\Sigma_{i_{1}}, \ldots, \Sigma_{i_{d}}\right)}{\partial\left(w_{1}, \ldots, w_{d}\right)}(\gamma) \not \equiv 0
$$

and

$$
\operatorname{ord}\left(\frac{\partial\left(\psi_{1}, \ldots, \psi_{d}\right)}{\partial\left(z_{i_{1}}, \ldots, z_{i_{d}}\right)}(\sigma \circ \gamma)\right) \leq d \mu
$$

Let

$$
\nu:=\operatorname{mult} f .
$$

Consider $(m+1)$-dimensional submanifold $L \subset \mathbb{C}^{m+d}$ passing through 0 such that mult $f$ equals the multiplicity of $\left.f\right|_{L}$ at 0 . Since the singular locus of $V$ is of codimension $\geq m+1$ in $\mathbb{C}^{m+d}$, we may assume

$$
L \cap V \subset V_{\text {reg }} \cup\{0\}
$$

and therefore $\widetilde{V} \cap \widetilde{L} \cap E$ is a finite set, where $\widetilde{L}$ is the strict transform of $L$ under $\sigma$. In fact, $\widetilde{V} \cap \widetilde{L} \cap E$ has at most $\nu$ elements. Since all smooth centers of $\sigma$ are disjoint from $V_{\text {reg }}$, we may further assume that $L$ intersects smooth centers only at 0 and only transversally and hence each connected component of $\widetilde{L}$ is smooth.

We claim that for $L$ with generic tangent space $T_{0} L, \widetilde{V} \cap \widetilde{L}$ is a disjoint union of at most $\nu$ smooth curves that intersect transversally with $\widetilde{V} \cap E$. Indeed, let $A$ be the space of all $\nu$-jets at 0 of all holomorphic curve germs $\gamma:(\mathbb{C}, 0) \rightarrow(\widetilde{M}, \gamma(0))$ such that $\gamma(0) \in \widetilde{V} \cap E$. Then $A$ is a subvariety in the jet space of all $\nu$-jets of holomorphic curves and the subset $B$ of all $\nu$-jets of curves $\gamma$ which are either singular or intersecting tangentially with $\widetilde{V} \cap E$ at $\gamma(0)$ is a proper subvariety. Let $p_{0}$ be a smooth point of $E \cap \widetilde{V}$. Choose a germ of a smooth curve $\gamma:(\mathbb{C}, 0) \rightarrow\left(\widetilde{V}, p_{0}\right)$ which intersects transversally with $\widetilde{V} \cap E$ at $p_{0}$, i.e. $j_{0}^{\nu} \gamma \in A \backslash B$. Since $\sigma \circ \gamma$ is a holomorphic curve in $V$ passing through 0 , we can choose a set of holomorphic functions $\phi_{j}, j=1, \ldots, d-1$, such that $\{f=\phi=0\}$ is 1-dimensional and

$$
\operatorname{image}(\sigma \circ \gamma) \subset\{f=\phi=0\},
$$

where $\phi=\left(\phi_{1}, \ldots, \phi_{d-1}\right)$. Then after a small perturbation of the differential of $\phi$, we may assume that $L=\{\phi=0\}$ is smooth and $\{f=\phi=0\}$ contains a curve $\sigma \circ \gamma$ with $j_{0}^{\nu} \gamma \in A \backslash B$, and all other components are 1-dimensional. Since $V$ is irreducible, any other component of $L \cap V$ can be connected with $\sigma \circ \gamma$ via paths in $V$. Hence any two components can be connected via a deformation of $\phi$ as regular function of its differential at 0 that can be used as parameter, proving the claim. 
Let $X$ be as before. Then $X$ is a proper subvariety of $\widetilde{V} \cap E$. Let $C$ be the subset of $A$ consisting of $\nu$-jets of curves $\gamma$ such that $\gamma(0) \in X$. Then $C$ is a proper subvariety of $A$. Since the union of all linear subspaces covers $\mathbb{C}^{m+d}$, there exists $L$ such that the $\nu$-jet at $\sigma^{-1}(0)$ of the lifting of a curve in $L \cap V$ under $\sigma$ is not contained in $C$. Let $\nu_{0} \leq \nu$ be the number of irreducible components of $V \cap L$ for generic linear subspace of codimension $d-1$. Then for a generic linear subspace $L$ of codimension $d-1$ we can define a map $j_{0}^{\nu}:\{L\} \rightarrow A \times \cdots \times A$ by

$$
j_{0}^{\nu}(L):=\left(j_{0}^{\nu} \gamma_{1}, \ldots, j_{0}^{\nu} \gamma_{\nu_{0}}\right)
$$

where $\cup_{j} \gamma_{j}$ is the lifting of $V \cap L$ under $\sigma$ such that

$$
\sigma\left(\gamma_{\ell}(0)\right)=0, \quad \ell=1, \ldots, \nu_{0}
$$

Since $B$ and $C$ are proper subvarieties of $A$, there exists $L$ such that

$$
j_{0}^{\nu} \gamma_{\ell} \in A \backslash(B \cup C), \quad \ell=1, \ldots, \nu_{0} .
$$

Therefore by the additivity of multiplicity, we obtain

$$
\operatorname{mult}\left(\left.f\right|_{L},\left.\frac{\partial\left(\psi_{1}, \ldots, \psi_{d}\right)}{\partial\left(z_{1}, \ldots, z_{d}\right)}\right|_{L}\right) \leq d \nu \mu
$$

for some coordinates at 0 constructed by a linear change of local coordinate systems satisfying (4.9) associated to irreducible components of $V \cap L$.

In the following corollary we use the convention that mult $(f)=1$ if $f$ has 0 components.

Corollary 4.2. Let $(f, \psi):\left(\mathbb{C}^{n}, 0\right) \rightarrow\left(\mathbb{C}^{n-d} \times \mathbb{C}^{d}, 0\right)$ be a holomorphic map germ satisfying

$$
\nu:=\operatorname{mult}(f)<\infty, \quad \mu:=\operatorname{mult}(f, \psi)<\infty .
$$

Then after a linear change of coordinates $\left(z_{1}, \ldots, z_{n}\right)$ and another linear coordinate change in $\mathbb{C}^{d}$, the partial Jacobian determinant

$$
J:=\frac{\partial\left(\psi_{1}, \ldots, \psi_{d}\right)}{\partial\left(z_{1}, \ldots, z_{d}\right)}
$$

satisfies

$$
\operatorname{mult}(f, J) \leq d \nu \mu, \quad \operatorname{mult}\left(f, J, \psi_{2}, \ldots, \psi_{d}\right) \leq d \nu \mu^{d}
$$

where $\psi_{j}$ is the $j$-th component of $\psi$ in the new coordinates.

Proof. Applying Proposition 4.1 to $\psi$, we conclude that the first inequality in (7.6) holds after a generic linear change of $z$. The second inequality is obtained by applying Lemma 2.4 to $(f, J)$ and $F:=(f, \psi)$. 


\section{EXISTENCE OF EFFECTIVE TRIANGULAR RESOLUTIONS}

The following is a more precise version of the meta-procedure (MP2) in Theorem 1.11;

Proposition 5.1. Let $1 \leq k \leq n, f_{1}, \ldots, f_{k}, \phi_{1}, \ldots, \phi_{n} \in \mathcal{O}_{n, 0}$, satisfy

$$
\mu_{j}:=\operatorname{mult}\left(f_{1}, \ldots, f_{j}, \phi_{j+1}, \ldots, \phi_{n}\right)<\infty, \quad 1 \leq j \leq k .
$$

Then the map germ $\phi=\left(\phi_{1}, \ldots, \phi_{n}\right)$ and the filtration of ideals $I_{j}:=\left(f_{1}, \ldots, f_{j}\right), 1 \leq j \leq k$, admit a triangular resolution $h=\left(h_{1}, \ldots, h_{k}\right)$ satisfying

$$
\operatorname{ord}_{w_{j}} h_{j} \leq n \cdot \mu_{j} \cdot \operatorname{mult}\left(f_{1}, \ldots, f_{j}\right), \quad 1 \leq j \leq k .
$$

In fact, each $h_{j}\left(w_{j}, \ldots, w_{n}\right)$ can be chosen as Weierstrass polynomial in $w_{j}$.

Proof. Consider the coordinate projections

$$
\pi_{j}\left(w_{1}, \ldots, w_{n}\right)=\left(w_{j}, \ldots, w_{n}\right) \in \mathbb{C}^{n-j+1}, \quad 1 \leq j \leq k
$$

and let

$$
W_{j}:=\mathcal{V}\left(f_{1}, \ldots, f_{j}\right), \quad \widetilde{W}_{j}:=\left(\pi_{j} \circ \phi\right)\left(W_{j}\right) \subset \mathbb{C}^{n-j+1}, \quad 1 \leq j \leq k,
$$

where $\mathcal{V}$ is the zero variety. Then $W_{j}$ is of codimension $\geq k$ in $\mathbb{C}^{n}$. In fact, counting preimages and using (7.1), we conclude that $\widetilde{W}_{j} \subset \mathbb{C}^{n-j+1}$ is a proper subvariety of codimension 1 and

$$
\left.\pi_{j+1}\right|_{\widetilde{W}_{j}}: \widetilde{W}_{j} \rightarrow \mathbb{C}^{n-j}
$$

is a finite holomorphic map germ of degree $\leq \mu_{j}$. Then there exist Weierstrass polynomials $Q_{j}\left(w_{j}, \ldots, w_{n}\right), j=1, \ldots, k$, satisfying

$$
Q_{j}=w_{j}^{\nu_{j}}+\sum_{\ell<\nu_{j}} b_{j, \ell}\left(w_{j+1}, \ldots, w_{n}\right) w_{j}^{\ell},\left.\quad Q_{j}\right|_{\widetilde{W}_{j}}=0, \quad \nu_{j}=\operatorname{ord}_{w_{j}} Q_{j} \leq \mu_{j} .
$$

Furthermore, Lemma 3.1 implies

$$
h_{j} \circ \phi \in\left(f_{1}, \ldots, f_{j}\right), \quad h_{j}:=Q_{j}^{\lambda_{j}},
$$

for suitable $\lambda_{j} \in \mathbb{N}_{\geq 1}$ satisfying

$$
\lambda_{j} \leq n \cdot \operatorname{mult}\left(f_{1}, \ldots, f_{j}\right) .
$$

Then $\left(h_{1}, \ldots, h_{k}\right)$ is a triangular resolution satisfying (5.2) as desired.

\section{EfFective Kohn's procedures For triangular RESOlutions}

The following is a more precise version of the meta-procedure (MP3) in Theorem 1.11:

Proposition 6.1. Let $1 \leq k<n$ and $\left(Q_{1}, \ldots, Q_{k+1}\right)$ be a triangular resolution of $(\Gamma, \mathcal{I})$, where $\Gamma:\left(\mathbb{C}^{n}, 0\right) \rightarrow\left(\mathbb{C}^{n}, 0\right)$ is a holomorphic map germ and $\mathcal{I}$ a filtration of ideals $I_{1} \subset \ldots \subset I_{k+1} \subset \mathcal{O}_{n, 0}$. Assume

$$
\mu_{j}=\operatorname{ord}_{w_{j}} Q_{j}<\infty, \quad 1 \leq j \leq k,
$$


and

$$
I_{k+1} \subset I_{k}+(J),
$$

where $J$ is the Jacobian determinant of $\Gamma$.

Then $Q_{k+1} \circ \Gamma$ can be obtained by applying holomorphic Kohn's procedures (P1) and (P2) to $\left(\psi, I_{k}\right)$ where $\psi=\left(\Gamma_{k+1}, \ldots, \Gamma_{n}\right)$ are the last $k$ components and each procedure $(P 1)$ and $(P 2)$ is applied $\mu_{1} \cdots \mu_{k}$ number of times with the root order in (P2) being $\leq k+1$. In particular, if $I_{k}$ consists of multipliers of order $\geq \varepsilon$, then $Q_{k+1} \circ \Gamma$ is a multiplier of order $\geq(2 k+2)^{-\mu_{1} \cdots \mu_{k} \varepsilon}$.

Proof. The proof is inspired by [S10, III.7], [S17, §3] and [CD10, §5]. Since $\mu_{j}<\infty$ for $j \leq k$, multiplying by invertible holomorphic functions, we may assume that

$$
Q_{j}=w_{j}^{\mu_{j}}+\sum_{\ell<\mu_{j}} b_{j, \ell}\left(w_{j+1}, \ldots, w_{n}\right) w_{j}^{\ell}, \quad 1 \leq j \leq k,
$$

are Weierstrass polynomials satisfying

$$
f_{j}:=Q_{j} \circ \Gamma \in I_{j}
$$

In addition, (6.2) implies

$$
f_{k+1}:=Q_{k+1} \circ \Gamma \in I_{k}+(J) .
$$

We use the reverse lexicographic order for $\mathbb{N}^{k}$, i.e. for $L=\left(\ell_{1}, \ldots, \ell_{k}\right)$ and $\widetilde{L}=\left(\tilde{\ell}_{1}, \ldots, \tilde{\ell}_{k}\right)$, we let $L<\widetilde{L}$ if there exists $j_{0}$ such that $\ell_{j}=\tilde{\ell}_{j}$ for $j>j_{0}$ and $\ell_{j_{0}}<\tilde{\ell}_{j_{0}}$. Let

$$
\Lambda:=\left\{L=\left(\ell_{1}, \ldots, \ell_{k}\right) \in \mathbb{Z}^{k}: 1 \leq \ell_{j} \leq \mu_{j}, j=1, \ldots, k\right\} .
$$

For $L \in \Lambda$, define $A_{L} \in \mathcal{O}_{n, 0}$ by

$$
A_{L}(w):=\partial_{w_{1}}^{\ell_{1}} Q_{1}(w) \cdots \partial_{w_{k}}^{\ell_{k}} Q_{k}(w)
$$

Since $f_{j} \in I_{j}$ by the definition of a triangular resolution,

$$
J_{(1, \ldots, 1)}:=\frac{\partial\left(f_{1}, \ldots, f_{k}, \psi_{k+1}, \ldots, \psi_{n}\right)}{\partial\left(z_{1}, \ldots, z_{n}\right),}
$$

which is the Jacobian determinant of the map

$$
\left(f_{1}, \ldots, f_{k}, \psi_{k+1}, \ldots, \psi_{n}\right)=\Phi \circ \Gamma
$$

where

$$
\Phi(w):=\left(Q_{1}(w), \ldots, Q_{k}(w), w_{k+1}, \ldots, w_{n}\right),
$$

Then the Jacobian factors as

$$
J_{(1, \ldots, 1)}=\left(A_{(1, \ldots, 1)} \circ \Gamma\right) J
$$

and hence by (6.3),

$$
\left(A_{(1, \ldots, 1)} \circ \Gamma\right) f_{k+1} \in I_{k}+\left(J_{(1, \ldots, 1)}\right)
$$

is obtained by applying Kohn's procedure (1) to $I_{k}$. 
Now for a given $L=\left(\ell_{1}, \ldots, \ell_{k}\right) \in \Lambda$ such that $L>(1, \ldots, 1)$, assume by induction that that $\left(A_{\widetilde{L}} \circ \Gamma\right) f_{k+1}$ is obtained by applying Kohn's procedures (P1) and (P2). Let

$$
m_{L}:=\#\left\{j: \ell_{j}>1\right\} \leq k
$$

be the number of $j \in\{1, \ldots, k\}$ with $\ell_{j}>1$. We will show that $\left(A_{L} \circ \Gamma\right) f_{k+1}$ is obtained by additionally applying once each of the Kohn's procedures (P1) and (P2) with the root order in (P2) being $\leq m_{L}+1 \leq k+1$. For $j=1, \ldots, k$, we define $B_{j}$ as follows:

(1) In the case $\ell_{j}>1$, set

$$
B_{j}:=\left(A_{L_{j}} \circ \Gamma\right) f_{k+1}=\left(A_{L_{j}} Q_{k+1}\right) \circ \Gamma
$$

with

$$
L_{j}:=\left(\mu_{1}, \ldots, \mu_{j-1}, \ell_{j}-1, \ell_{j+1}, \ldots, \ell_{k}\right)<L .
$$

(2) In the remaining case $\ell_{j}=1$, set

$$
B_{j}:=Q_{j} \circ \Gamma=f_{j} .
$$

By our assumption, $B_{j}$ in both cases are obtained by applying Kohn's procedures.

Now apply (1) to obtain

$$
J_{L}:=\frac{\partial\left(B_{1}, \ldots, B_{k}, \Gamma_{k+1}, \ldots, \Gamma_{n}\right)}{\partial\left(z_{1}, \ldots, z_{n}\right)} .
$$

Then $J_{L}$ is the Jacobian determinant of the map

$$
\left(B_{1}, \ldots, B_{k}, \Gamma_{k+1}, \ldots, \Gamma_{n}\right)=\Phi \circ \Gamma,
$$

with $\Phi$ as in (6.4). In view of our assumption that each $Q_{j}, j \leq k$ is a Weierstrass polynomial in $w_{j}$ of degree $\mu_{j}$, each top derivative $\partial_{w_{j}}^{\mu_{j}} Q_{j}$ is constant and hence $B_{j}$ only depends on $\left(w_{j}, \ldots, w_{n}\right)$. Then using factorization of the Jacobian determinant and the triangular property of $B_{j}$ 's, we obtain

$$
J_{L}=c\left(\left(\left(\partial_{w_{1}}^{\ell_{1}} Q_{1}\right)^{m_{1}} \cdots\left(\partial_{w_{k}}^{\ell_{k}} Q_{k}\right)^{m_{k}} Q_{k+1}^{m_{L}}\right) \circ \Gamma\right) J
$$

for some constant $c \neq 0$ and integers $m_{j} \leq m_{L}+1$, and hence by (6.3),

$$
\left(\left(A_{L} Q_{k+1}\right) \circ \Gamma\right)^{m_{L}+1} \in I_{k}+\left(J_{L}\right) .
$$

Then $\left(A_{L} Q_{k+1}\right) \circ \Gamma$ is obtained by the Kohn's procedure $(2)$ with root order $\leq m_{L}+1 \leq k+1$, and the proof is complete by induction.

\section{EFFeCtive estimates}

Corollary 7.1. Let $0 \leq k<n \leq N$ and $F_{1}, \ldots, F_{N}, f_{1}, \ldots, f_{k} \in \mathcal{O}_{n, 0}$, satisfy

$$
\nu:=\operatorname{mult}\left(F_{1}, \ldots, F_{N}\right)<\infty, \quad \mu:=\operatorname{mult}\left(f_{1}, \ldots, f_{k}\right)<\infty,
$$

(where $\mu:=1$ in case $k=0)$. Then there exists a $(k+1)$-tuple $g_{1}, \ldots, g_{k+1} \in \mathcal{O}_{n, 0}$ with

$$
\text { mult }\left(g_{1}, \ldots, g_{k+1}\right) \leq n^{k+3} \mu^{n+k+3} \nu^{(n-k)(n+1)},
$$


where $g_{j} \in\left(f_{1}, \ldots, f_{j}\right)$ for $1 \leq j \leq k$, and $g_{k+1}$ is obtained from the initial set $\left\{F_{1}, \ldots, F_{N}\right\}$ and the ideal $\left(f_{1}, \ldots, f_{k}\right)$ via the Kohn's procedures (P1) and (P2), each applied $\leq\left(n \mu^{2} \nu^{n-k}\right)^{k}$ number of times with the root order in (PQ) being $\leq k+1$. In particular, if $f_{1}, \ldots, f_{k}$ are multipliers of order at least $\varepsilon>0$, then $g_{k+1}$ is a multiplier of order

$$
\geq \frac{\varepsilon}{(2 k+2)^{\left(n \mu^{2} \nu^{n-k}\right)^{k}}} \text {. }
$$

Furthermore, after a linear change of coordinates $z=\left(z_{1}, \ldots, z_{n}\right), g_{j}$ can be chosen of the form

$$
g_{j}(z)=Q_{j}\left(z_{j}, \ldots, z_{k}, \psi_{k+1}(z), \ldots, \psi_{n}(z)\right), \quad 1 \leq j \leq k+1,
$$

where $\psi_{j}$ 's are linear combinations of $F_{j}$ 's.

Proof. Applying Lemma 2.4, we obtain (generic) linear combinations $\psi=\left(\psi_{k+1}, \ldots, \psi_{n}\right)$ of $F_{j}$ 's such that

$$
\operatorname{mult}\left(z_{1}, \ldots, z_{k}, \psi\right) \leq \nu^{n-k}, \quad \operatorname{mult}(f, \psi) \leq \mu \nu^{n-k} .
$$

Next apply Corollary 4.2 to obtain, after (generic) linear change of coordinates $z=\left(z_{1}, \ldots, z_{n}\right)$ and (generic) linear transformation of $\psi_{j}$ 's,

$$
\text { mult }(f, J) \leq n \cdot \operatorname{mult}(f) \cdot \operatorname{mult}(f, \psi) \leq n \mu^{2} \nu^{n-k},
$$

and

$$
\operatorname{mult}\left(f, J, \psi_{k+2}, \ldots, \psi_{n}\right) \leq n \cdot \operatorname{mult}(f) \cdot(\operatorname{mult}(f, \psi))^{n-k} \leq n \cdot \mu\left(\mu \nu^{n-k}\right)^{n-k}
$$

where

$$
J:=\frac{\partial\left(\psi_{k+1}, \ldots, \psi_{n}\right)}{\partial\left(z_{k+1}, \ldots, z_{n}\right)} .
$$

Now apply Proposition 5.1 for the map germ

$$
\Gamma(z):=\left(z_{1}, \ldots, z_{k}, \psi(z)\right),
$$

and the filtration of ideals

$$
I_{j}:=\left(f_{1}, \ldots, f_{j}\right), 1 \leq j \leq k, \quad I_{k+1}:=\left(f_{1}, \ldots, f_{k}, J\right),
$$

to obtain a triangular resolution $\left(h_{1}, \ldots, h_{k+1}\right)$ satisfying

$$
\operatorname{ord}_{w_{j}} h_{j} \leq n \cdot \operatorname{mult}(f) \cdot \operatorname{mult}(f, \psi) \leq n \mu^{2} \nu^{n-k}
$$

for $1 \leq j \leq k$ and

$$
\begin{aligned}
& \operatorname{ord}_{w_{j+1}} h_{k+1} \leq n \cdot \operatorname{mult}(f, J) \cdot \operatorname{mult}\left(f, J, \psi_{k+2}, \ldots, \psi_{n}\right) \\
& \quad \leq n \cdot n \mu^{2} \nu^{n-k} \cdot n \cdot \mu\left(\mu \nu^{n-k}\right)^{n-k} \leq n^{3} \mu^{n-k+3} \nu^{(n-k)(n-k+1)},
\end{aligned}
$$

in view of (7.5) and (7.6). Setting

$$
g_{j}:=h_{j} \circ \Gamma, \quad 1 \leq j \leq k+1,
$$


and counting preimages using the multiplicativity of the multiplicity under composition, we obtain

$$
\text { mult }\left(g_{1}, \ldots, g_{k+1}\right) \leq \operatorname{ord}_{w_{1}} h_{1} \cdots \operatorname{ord}_{w_{k+1}} h_{k+1} \cdot \operatorname{mult}(\Gamma)
$$

implying

$$
\operatorname{mult}\left(g_{1}, \ldots, g_{k+1}\right) \leq\left(n \mu^{2} \nu^{n-k}\right)^{k} \cdot n^{3} \mu^{3+n-k} \nu^{(n-k)(n-k+1)} \cdot \nu^{n-k}=n^{k+3} \mu^{n+k+3} \nu^{(n-k)(n+1)} .
$$

Since $J$ equals the Jacobian determinant of $\Gamma$ and $I_{k+1} \subset I_{k}+(J)$, we can apply Proposition 6.1 to conclude that $g_{k+1}$ can be obtained by applying holomorphic Kohn's procedures to the initial set of components $\psi$ and the ideal $I_{k}$ with each procedure (P1) and (P2) applied

$$
\operatorname{ord}_{w_{1}} h_{1} \cdots \operatorname{ord}_{w_{k}} h_{k} \leq\left(n \mu^{2} \nu^{n-k}\right)^{k}
$$

number of times with the root order in (2) being $\leq k+1$.

In particular, if each $\psi_{j}$ is a pre-multiplier and each $f_{j}$ a multiplier of order $\geq \varepsilon$, it follows that each $g_{j} \in I_{j}=\left(f_{1}, \ldots, f_{j}\right)$ is a multiplier of order $\geq \varepsilon$ for $1 \leq j \leq k$ and $g_{k+1}$ is a multiplier of order (7.3) as desired.

Corollary 7.2. There exists a function $\varepsilon: \mathbb{N}_{>0} \times \mathbb{N}_{>0} \rightarrow \mathbb{R}_{>0}$, such that for every collection of holomorphic pre-multipliers in $\mathbb{C}^{n}$ with multiplicity $\leq \nu$, there is a finite sequence of Kohn's procedures (1) and (2) in Definition 1.2 producing the unit as a multiplier of order $\geq \varepsilon(n, \nu)$. In fact, one can take

$$
\varepsilon(n, \nu)=\frac{1}{4(2 n+2)^{2(n \nu)^{(3 n)^{n+1}}} .}
$$

Proof. The case $n=1$ is well-known: for any premultiplier $\psi$ of multiplicity $\leq \nu$, the unit is a multiplier of order $\geq 1 / 4 \nu$, which is greater than $\varepsilon$ given by (7.9) with $n=1$.

Thus we may assume $n \geq 2$ in the following. Applying Corollary 7.1 repeatedly starting from $k=0$, we obtain holomorphic function germs

$$
f_{k, j} \in \mathcal{O}_{n, 0}, \quad 1 \leq j \leq k \leq n
$$

with

$$
\operatorname{mult}\left(f_{k, 1}, \ldots, f_{k, k}\right) \leq \mu_{k},
$$

where $\mu_{k}$ are given recursively by

$$
\mu_{0}=1, \quad \mu_{k+1}=n^{k+3} \nu^{(n-k)(n+1)} \mu_{k}^{n+k+3},
$$

from which

$$
\mu_{k}=n^{a_{k}} \nu^{b_{k}}
$$

where the integer sequences $\left(a_{k}\right)$ and $\left(b_{k}\right)$ are given recursively by

$a_{1}=3, \quad b_{1}=n(n+1), \quad a_{k+1}=(n+k+3) a_{k}+k+3, \quad b_{k+1}=(n+k+3) b_{k}+(n-k)(n+1)$.

Since $n \geq 1$, it is easy to see by induction that $a_{k} \geq k+3$, from which it follows that

$$
a_{k+1} \leq(n+k+3) a_{k}+a_{k}=(n+k+4) a_{k}, \quad k \geq 1,
$$


and then by induction

$$
a_{k+1} \leq(n+5)(n+6) \cdots(n+k+4) a_{1} \leq 3(2 n+3)^{k} \leq(3 n)^{k+1}, \quad n \geq 3,
$$

while for $n=2$, the same inequality can be checked directly:

$$
a_{2} \leq 3(2+5) \leq(3 \cdot 2)^{2} .
$$

Similarly,

$$
b_{k+1} \leq(n+k+3) b_{k}+b_{k}=(n+k+4) b_{k},
$$

whence

$$
b_{k+1} \leq(n+5) \cdots(n+k+4) b_{1} \leq n(n+1)(2 n+3)^{k} \leq(3 n)^{k+2}, \quad n \geq 3,
$$

and directly for $n=2$ :

$$
b_{2} \leq(2+5) \cdot 2(2+1) \leq(3 \cdot 2)^{3} .
$$

Putting all together, we obtain

$$
\mu_{k} \leq n^{(3 n)^{k}} \nu^{(3 n)^{k+1}} .
$$

Next starting with $\varepsilon=1 / 2$ and iterating (7.3), we conclude that $f_{k, j}$ are multipliers of order $\geq \varepsilon_{k}$, where

$$
\varepsilon_{0}=1 / 2, \quad \varepsilon_{k+1}=\frac{\varepsilon_{k}}{(2 k+2)^{\left(n \mu_{k}^{2} \nu^{n-k}\right)^{k}}}
$$

hence

$$
\varepsilon_{k+1} \geq \frac{1}{2(2 n+2)^{A_{k+1}}}
$$

where

$$
A_{k+1}=\sum_{j=1}^{k}\left(n \nu^{n-j} \mu_{j}^{2}\right)^{j} \leq k\left(n \nu^{n-k} \mu_{k}^{2}\right)^{k} \leq(n-1)\left(n \nu^{n-1} \cdot n^{2(3 n)^{k}} \nu^{2(3 n)^{k+1}}\right)^{k} \leq(n \nu)^{(3 n)^{k+2}},
$$

and finally

$$
\varepsilon_{n} \geq \frac{1}{2(2 n+2)^{(n \nu)^{(3 n)^{n+1}}}}
$$

Now consider the monomials

$$
z_{j}^{\mu_{n}} \in\left(f_{n, 1}, \ldots, f_{n, n}\right)
$$

and apply Kohn's procedure (2) to conclude that each $z_{j}$ is a multiplier of order $\varepsilon_{n} / \mu_{n}$, and applying procedure (1), we conclude that 1 is a multiplier of order

$$
\frac{\varepsilon_{n}}{2 \mu_{n}} \geq \frac{1}{4(2 n+2)^{(n \nu)^{(3 n)^{n+1}}}(n \nu)^{(3 n)^{n+1}}} \geq \frac{1}{4(2 n+2)^{2(n \nu)^{(3 n)^{n+1}}} .}
$$




\section{EXAMPles of THE EFFECTIVE ALGORITHM}

We shall consider here classes of special domains in $\mathbb{C}^{4}$ locally given by

$$
\operatorname{Im} z_{4}+\sum_{j=1}^{3}\left|\psi_{j}\left(z_{1}, z_{2}, z_{3}\right)\right|^{2}<0,
$$

where

$$
\psi_{j}(z)=z_{j}^{2}+\ldots, \quad j=1,2,3, \quad z \in \mathbb{C}^{3},
$$

where we use the following convention:

Convention. The dots stand for arbitrary terms of order higher than all previous terms.

We shall perform our analysis in a neighborhood of 0 and hence regard all functions as germs at 0 . The type (at 0 ) of $\psi=\left(\psi_{1}, \psi_{2}, \psi_{3}\right)$ is 2 and the multiplicity

$$
\mu:=\operatorname{mult}\left(\psi_{1}, \psi_{2}, \psi_{3}\right)=2^{3}=8 .
$$

In course of our effective algorithmic process, we repeatedly apply linear changes of coordinates $z=\left(z_{1}, z_{2}, z_{3}\right)$, while it will be convenient to rename and keep the old coordinates as $u=\left(u_{1}, u_{2}, u_{3}\right)$.

Step 0: The first multiplier - select a Jacobian determinant of pre-multipliers. In general we select pre-multipliers among arbitrary linear combinations of the $\psi_{j}$ with constant coefficients. (Note that combination with holomorphic coefficients may not be pre-multipliers). In our case the Jacobian ideal is generated by the single function

$$
J_{1}(z)=\frac{\partial\left(\psi_{1}, \psi_{2}, \psi_{3}\right)}{\partial\left(z_{1}, z_{2}, z_{3}\right)}(z) \sim z_{1} z_{2} z_{3}+\ldots, \quad \text { mult } J_{1}=3,
$$

where we generally write $\sim$ for an equality up to an invertible germ of holomorphic function.

Step 1.1: Select generic complementary pre-multipliers. Following Siu [S10, (III.3)], we look for pre-multipliers $\phi_{2}, \phi_{3}$ among generic linear combinations of $\psi_{j}$ complementing $J_{1}$ in the sense of an effective joint multiplicity, i.e. such that mult $\left(J_{1}, \phi_{2}, \phi_{3}\right)$ is effectively bounded. In our case, we can replace $\psi_{j}$ with combinations

$$
\psi_{j}:=z_{j}^{2}-z_{1}^{2}+\ldots, \quad j=2,3,
$$

that yield the effective bound

$$
\operatorname{mult}\left(J_{1}, \psi_{2}, \psi_{3}\right)=\operatorname{dim} \mathcal{O}_{3} /\left(J_{1}, \psi_{2}, \psi_{3}\right) \leq 5 \cdot 2 \cdot 2=20 .
$$

Indeed

$$
\left(z_{2} z_{3}\right) J_{1} \sim z_{1} z_{2}^{2} z_{3}^{2}+\ldots=z_{1}^{5}+\ldots \bmod \left(\psi_{2}, \psi_{3}\right),
$$

and hence the quotient in (8.2) is spanned by the monomials

$$
z_{1}^{\alpha_{1}} z_{2}^{\alpha_{2}} z_{3}^{\alpha_{3}}, \quad \alpha_{1} \leq 4, \quad \alpha_{2}, \alpha_{3} \leq 1 .
$$


Step 1.2: Select a direction of an effective vanishing order. We next look to change the linear coordinates $\left(z_{1}, z_{2}, z_{3}\right)$ to make effectively bounded the vanishing order of $J$ along $z_{2}=z_{3}=0$ and the multiplicity mult $\left(z_{1}, \psi_{2}, \psi_{3}\right)$. For this, consider coordinate change with

$$
\left(u_{1}, u_{2}, u_{3}\right)=\left(z_{1}, z_{2}+z_{1}, z_{3}+z_{1}\right)
$$

so that our data take the form

$$
J \sim z_{1}\left(z_{2}+z_{1}\right)\left(z_{3}+z_{1}\right)+\ldots=u_{1} u_{2} u_{3}+\ldots
$$

along with

$$
\psi_{j}=\left(z_{j}+z_{1}\right)^{2}-z_{1}^{2}+\ldots=u_{j}^{2}-u_{1}^{2}+\ldots
$$

and

$$
\operatorname{mult}\left(\Gamma_{1}\right)=2^{2}=4, \quad \Gamma_{1}(z):=\left(z_{1}, \psi_{2}(z), \psi_{3}(z)\right),
$$

where $\Gamma_{1}$ is the finite map as in Corollary 1.12 that is used to decompose multipliers.

Step 1.3: Select a decomposable multiplier $f_{1}=Q \circ \Gamma_{1}$ in the radical of $(J)$. We look for a multiplier $f_{1}=Q \circ \Gamma_{1}$ as in Corollary 1.12, where $Q$ is a holomorphic function with effectively bounded vanishing order $\mu_{1}$ in $z_{1}$ :

$$
\mu_{1} \leq n \mu^{n}=3 \cdot 8^{3}
$$

Any method can be used to construct $f_{1}$ here. One possible approach consists of taking $f_{1}$ to be the product of all transformations of $J$ under the action of the deck transformation group of the finite covering map germ $\Gamma_{1}:\left(\mathbb{C}^{n}, 0\right) \rightarrow\left(\mathbb{C}^{n}, 0\right)$, i.e. setting

$$
f_{1}:=\prod_{g \in \operatorname{Deck}\left(\Gamma_{\psi}\right)} J \circ g
$$

where $\operatorname{Deck}\left(\Gamma_{1}\right)$ denotes the group of all deck transformations, i.e. germs of biholomorphic transformations $g:\left(\mathbb{C}^{n}, 0\right) \rightarrow\left(\mathbb{C}^{n}, 0\right)$ with $\Gamma_{1} \circ g=\Gamma_{1}$.

In our case, the deck transformation group of $\Gamma_{1}$ is generated by the involutions changing the signs of some of $u_{j}, j=2,3$, i.e. transformations having in $u$-coordinates the form

$$
g(z)=\left(z_{1}, \varepsilon_{2}\left(z_{2}+z_{1}\right)-z_{1}, \varepsilon_{3}\left(z_{n}+z_{1}\right)-z_{1}\right)+\ldots, \quad \varepsilon_{2}, \varepsilon_{3} \in\{1,-1\} .
$$

Then from (8.3) we obtain

$$
f_{1} \sim\left(z_{1}\left(z_{2}+z_{1}\right)\left(z_{3}+z_{1}\right)\right)^{4}+\ldots=\left(u_{1} u_{2} u_{3}\right)^{4}+\ldots,
$$

where

$$
\operatorname{mult}\left(f_{1}, \psi_{2}, \psi_{3}\right) \leq n \mu^{n+1}=3 \cdot 8^{4}
$$


Step 2.1: Select a complementary partial determinant. We next follow the lines of the proof of Corollary 7.1 with $k=1$ and look for a linear change of coordinates such that, for the partial determinant

$$
J_{2}=\frac{\partial\left(\psi_{2}, \psi_{3}\right)}{\partial\left(z_{2}, z_{3}\right)}
$$

the multiplicity

$$
\operatorname{mult}\left(f_{1}, J_{2}, \psi_{3}\right)
$$

is effectively bounded. In general, the existence of such coordinates follows from Corollary 4.2. In our case, we can apply a coordinate change with

$$
\left(u_{1}, u_{2}, u_{3}\right)=\left(z_{1}+z_{2}+z_{3}, z_{2}+z_{1}, z_{3}+z_{1}\right)
$$

and hence transforming our data into

$$
\psi_{j}=\left(z_{j}+z_{1}\right)^{2}-\left(z_{1}+z_{2}+z_{3}\right)^{2}+\ldots
$$

and

$$
f_{1} \sim\left(\left(z_{1}+z_{2}+z_{3}\right)\left(z_{1}+z_{2}\right)\left(z_{1}+z_{3}\right)\right)^{4}+\ldots
$$

Then computing (8.4) yields:

$$
J_{2}=\left(z_{2}+z_{1}\right)\left(z_{3}+z_{1}\right)-\left(2 z_{1}+z_{2}+z_{3}\right)\left(z_{1}+z_{2}+z_{3}\right)+\ldots,
$$

or equivalently

$$
J_{2}=u_{2} u_{3}-\left(u_{2}+u_{3}\right) u_{1}+\ldots
$$

Step 2.2: Select complementary pre-multipliers. By now we have an effectively bounded multiplicity mult $\left(f_{1}, J_{2}\right)$. In order to effectively bound (8.5), we replace $\psi_{3}$ with a linear combination of $\psi_{j}$, denoted again by $\psi_{3}$ by a slight abuse of notation,

$$
\psi_{3}:=u_{1}^{2}+u_{2}^{2}+u_{3}^{2}+\ldots
$$

Indeed, in the $u$-coordinates we have

$$
f_{1} \sim\left(u_{1} u_{2} u_{3}\right)^{4}+\ldots,
$$

and it is easy to verify that the lowest order terms of $\left(f_{1}, J_{2}, \psi_{3}\right)$ have no common zeroes other than $z=0$ and

$$
\operatorname{mult}\left(f_{1}, J_{2}\right) \leq \operatorname{mult}\left(f_{1}, J_{2}, \psi_{3}\right) \leq n\left(\mu_{1} \mu\right)^{n}=3 \cdot\left(8 \mu_{1}\right)^{3} \text {. }
$$


Step 2.3: Select decomposable function $H=Q_{2} \circ \Gamma_{1}$ in the radical of the ideal $\left(f_{1}, J_{2}\right)$. We look for a function $H=Q_{2} \circ \Gamma_{1}$ as in Proposition 6.1, where $Q_{2}$ is a Weierstrass polynomial of effectively bounded degree in $w_{2}$ with holomorphic coefficients in $w_{3}$. For our purposes, it will suffice to select $Q_{2}\left(w_{2}, w_{3}\right)$ to be just holomorphic with an effectively bounded vanishing order in $w_{2}$

$$
\operatorname{ord}_{w_{2}} Q_{2}=\nu \leq n\left(\mu \mu_{1}\right)^{n} .
$$

Then using Proposition 6.1, we conclude that $H$ is a multiplier of an order effectively bounded from below.

Step 2.4: Select new decomposable multipliers. Proceeding as in the proof of Corollary 7.1 , we take

$$
\Gamma_{2}(z):=\left(z_{1}, z_{2}, \psi_{3}(z)\right),
$$

and look for the multipliers of the form

$$
\widetilde{f}_{j}=\widetilde{Q}_{j} \circ \Gamma_{2}, \quad j=1,2,
$$

such that

$$
\nu_{1}=\operatorname{mult}\left(\widetilde{Q}_{1}\left(w_{1}, w_{2}, w_{3}\right), w_{2}, w_{3}\right) \leq \mu \mu_{1} \quad \nu_{2}=\operatorname{mult}\left(\widetilde{Q}_{2}\left(w_{2}, w_{3}\right), w_{3}\right) \leq n\left(\mu \mu_{1}\right)^{n+1} .
$$

Step 3.1: Select complementary partial determinant. We now repeat the process with $k=2$ in the proof of Corollary 7.1. We have

$$
\operatorname{mult}(f) \leq \operatorname{mult}(f, \widetilde{\psi}) \leq \mu \nu_{1} \nu_{2} \leq n \mu\left(\mu \mu_{1}\right)^{n+2}
$$

where $f=\left(\tilde{f}_{1}, \tilde{f}_{2}\right)$. Taking again suitable linear change of coordinates $z$, we may assume as in Corollary 4.2 that

$$
\operatorname{mult}\left(f, J_{3}\right) \leq \operatorname{mult}(f) \operatorname{mult}(f, \widetilde{\psi}) \leq(n \mu)^{2}\left(\mu \mu_{1}\right)^{2 n+4},
$$

where

$$
J_{3}=\frac{\partial \psi_{3}}{\partial z_{3}}
$$

Step 3.2: Select a decomposable function in the radical of the ideal $\left(f, J_{3}\right)$. Note that there is no analogue for Step 2.2 because $\left(f, J_{3}\right)$ is finite. Next, similarly to Step 2.3, select $\widetilde{H}=\widetilde{Q}_{3} \circ \Gamma_{2}$, where $\widetilde{Q}_{3}=\widetilde{Q}_{3}\left(w_{3}\right)$ has multiplicity effectively bounded by

$$
\operatorname{ord}_{w_{3}} \widetilde{Q}_{3} \leq n\left(\mu \nu_{1} \nu_{2}\right)^{n} \leq n\left(n \mu\left(\mu \mu_{1}\right)^{n+2}\right)^{n}=n^{n+1} \mu^{n}\left(\mu \mu_{1}\right)^{n^{2}+2 n} .
$$

Then $\widetilde{H}$ is multiplier with effectively bounded order by Proposition 6.1. 
Step 3.3: Effective termination. We obtain the multipliers

$$
\left(\widetilde{f}_{1}, \widetilde{f}_{2}, \widetilde{H}\right)=\left(\widetilde{Q}_{1}, \widetilde{Q}_{2}, \widetilde{Q}_{3}\right) \circ \Gamma_{2}
$$

and

$$
\operatorname{mult}\left(\widetilde{f}_{1}, \widetilde{f}_{2}, \widetilde{H}\right) \leq \operatorname{mult}\left(\widetilde{Q}_{1}, \widetilde{Q}_{2}, \widetilde{Q}_{3}\right) \cdot \operatorname{mult}\left(\Gamma_{2}\right) \leq \nu_{1} \nu_{2}\left(\operatorname{ord}_{w_{3}} \widetilde{Q}_{3}\right) \cdot \mu .
$$

Thus we have constructed a triple of multipliers of effective subellipticity orders with finite effectively bounded multiplicity. Hence the linear coordinate functions $z_{j}$ are in the effective radical of $\left(\widetilde{f}_{1}, \widetilde{f}_{2}, \widetilde{H}\right)$ and hence the algorithm terminates by taking the Jacobian of $\left(z_{1}, z_{2}, z_{3}\right)$, which is a multiplier with an effectively bounded subellipticity order. All effective bounds are directly computable from the above estimates similarly to Corollary 7.2 .

\section{REFERENCES}

[AGV85] Arnold V.I.; Gusein-Zade S.M.; Varchenko A.N. Singularities of Differentiable Maps. Monographs in Mathematics, vol 82. Birkhäuser Boston, 1985.

[BHR96] Baouendi, M.S.; Huang, X.; Rothschild, L.P. Regularity of CR mappings between algebraic hypersurfaces. Inventiones mathematicae, 125, 13-36 (1996).

[BS92] Boas, H.P.; Straube, E.J. On equality of line type and variety type of real hypersurfaces in $\mathbb{C}^{n}$. J. Geom. Anal. 2 (1992), no. 2, 95-98.

[BN15] Brinzanescu, V.; Nicoara, A.C. On the relationship between D'Angelo $q$-type and Catlin q-type. J. Geom. Anal., 25(3):17011719, 2015. Correction: J. Geom. Anal., 2019. https://doi.org/10.1007/s12220-019-00176-5

[BN19] Brinzanescu, V.; Nicoara, A.C. Relating Catlin and D'Angelo q-types Preprint 2019. https://arxiv.org/abs/1707.08294

[CD10] Catlin, D.W.; D'Angelo, J.P. Subelliptic estimates. Complex analysis, 75-94, Trends Math., Birkhäuser/Springer Basel AG, Basel, 2010.

[Ch06] Cho, J.-S. An algebraic version of subelliptic multipliers. Michigan Math. J. 54 (2006), no. 2, 411-426.

[D79] D'Angelo, J.P. Finite type conditions for real hypersurfaces. J. Differential Geom. 14 (1979), no. 1, 59-66 (1980).

[D82] D'Angelo, J.P. Real hypersurfaces, orders of contact, and applications. Ann. of Math. (2), 115 (3), 615637, 1982.

[D93] D'Angelo, J.P. Several complex variables and the geometry of real hypersurfaces. Studies in Advanced Mathematics. CRC Press, Boca Raton, FL, 1993.

[D95] D'Angelo, J.P. Finite type conditions and subelliptic estimates. Modern methods in complex analysis (Princeton, NJ, 1992), 63-78, Ann. of Math. Stud., 137, Princeton Univ. Press, Princeton, NJ, 1995.

[D17] D'Angelo, J.P. A Remark on Finite Type Conditions. J. Geom. Anal. 28, 2602-2608 (2018).

[DK99] D'Angelo, J.P.; Kohn, J.J. Subelliptic estimates and finite type. Several complex variables (Berkeley, CA, 1995-1996), 199-232, Math. Sci. Res. Inst. Publ., 37, Cambridge Univ. Press, Cambridge, 1999.

[DF79] Diederich, K.; Fornæss, J.E. Proper holomorphic maps onto pseudoconvex domains with real-analytic boundary. Ann. of Math. (2) $\mathbf{1 1 0}$ (1979), no. 3, 575-592.

[ELT77] Eisenbud, D.; Levine, H.I. and Teissier, B. An Algebraic Formula for the Degree of a $C^{\infty}$ Map Germ / Sur une inégalité à la Minkowski pour les multiplicités. Annals of Mathematics, Second Series, Vol. 106, No. 1 (Jul., 1977), pp. 19-44.

[Fa19] Fassina, M. A remark on two notions of order of contact. J. Geom. Anal. 29 (2019), no. 1, 707-716.

[Fa20] Fassina, M. Singularities And Multiplier Algorithms For Real Hypersurfaces. Dissertation. Urbana, Illinois, 2020. 
[FLZ14] Fornæss, J.E.; Lee, L.; Zhang, Y. Formal complex curves in real smooth hypersurfaces. Illinois J. Math. 58 (2014), no. 1, 1-10.

[FM94] Fornæss, J.E.; McNeal, J.D. A construction of peak functions on some finite type domains. Amer. J. Math. 116 (1994), no. 3, 737-755.

[FIK96] Fu, S.; Isaev, A.V.; Krantz, S.G. Finite type conditions on Reinhardt domains. Complex Variables Theory Appl. 31 (1996), no. 4, 357-363.

[Fu84] Fulton, W. Introduction to Intersection Theory in Algebraic Geometry. CBMS Regional Conference Series in Mathematics. Volume: 54; 1984; 83 pp.

[GGP19] García Barroso, E. R., González Pérez, P. D., Popescu-Pampu, P. How Newton polygons blossom into lotuses. Preprint 2019. https://arxiv.org/abs/1909.06974

[He08] Heier, G. Finite type and the effective Nullstellensatz. Comm. Algebra 36 (2008), no. 8, $2947-2957$. https://arxiv.org/abs/math/0603666

[Ho65] Hörmander, L. $L^{2}$-estimates and existence theorems for the $\bar{\partial}$-operator, Acta Math. 113 (1965), 89-152.

[HY19] Huang, X.; Yin, W. Regular multi-types and the Bloom conjecture. Preprint 2019. https://arxiv.org/abs/1902.10581

[KaK12] Kaveh, K.; Khovanskii, A.G. Newton-Okounkov bodies, semigroups of integral points, graded algebras and intersection theory. Annals of Mathematics, 176 (2012), 925-978.

[KZ18] Kim, S.Y.; Zaitsev, D. Jet vanishing orders and effectivity of Kohn's algorithm in dimension 3. Asian Journal of Mathematics. 22 (2018), no. 3, 545-568. Special issue in honor of Ngaiming Mok. https://arxiv.org/abs/1702.06908

[K72] Kohn, J. J. Boundary behavior of $\partial$ on weakly pseudo-convex manifolds of dimension two. Collection of articles dedicated to S. S. Chern and D. C. Spencer on their sixtieth birthdays. J. Differential Geometry 6 (1972), 523-542.

[Ko79] Kohn, J. J. Subellipticity of the $\bar{\partial}$-Neumann problem on pseudo-convex domains: sufficient conditions. Acta Math. 142 (1979), no. 1-2, 79-122. https://projecteuclid.org/euclid.acta/1485890016

[KoN65] Kohn, J. J.; Nirenberg, L. Non-coercive boundary value problems. Comm. Pure Appl. Math. 18 (1965), $443-492$.

[Ko04] Kohn, J.J. Ideals of multipliers. Complex analysis in several variables - Memorial Conference of Kiyoshi Oka's Centennial Birthday, 147-157, Adv. Stud. Pure Math., 42, Math. Soc. Japan, Tokyo, 2004.

[LM09] Lazarsfeld, R.; Mustata, M. Convex bodies associated to linear series Ann. Scient. Éc. Norm. Sup., 4e série, t. 42, 2009, 783-835.

[LJT08] Lejeune-Jalabert, M.; Teissier, B. Clôture intégrale des idéaux et équisingularité. Annales de la faculté des sciences de Toulouse Sér. 6, 17, no. 4 (2008), p. 781-859.

[M92] McNeal, J.D. Lower bounds on the Bergman metric near a point of finite type. Ann. of Math. (2) 136 (1992), no. 2, 339-360.

[MM17] McNeal, J.D., Mernik, L. Regular versus singular order of contact on pseudoconvex hypersurfaces. $J$ Geom Anal 28, 2653-2669 (2018).

[N14] Nicoara, A.C. Direct Proof of Termination of the Kohn Algorithm in the Real-Analytic Case. Preprint 2014. https://arxiv.org/abs/1409.0963

[O96] Okounkov, A. Brunn-Minkowski inequality for multiplicities, Invent. Math. 125 (1996), no. 3, $405-411$.

[RS76] Rothschild, L.P.; Stein, E.M. Hypoelliptic differential operators and nilpotent groups. Acta Math. 137 (1976), no. 3-4, 247-320.

[S01] Siu, Y.-T. Very ampleness part of Fujita's conjecture and multiplier ideal sheaves of Kohn and Nadel. Complex analysis and geometry (Columbus, OH, 1999), 171-191, Ohio State Univ. Math. Res. Inst. Publ., 9, de Gruyter, Berlin, 2001. 
[S02] Siu, Y.-T. Some recent transcendental techniques in algebraic and complex geometry. Proceedings of the International Congress of Mathematicians, Vol. I (Beijing, 2002), 439-448, Higher Ed. Press, Beijing, 2002.

[S05] Siu, Y.-T. Multiplier ideal sheaves in complex and algebraic geometry. Sci. China Ser. A 48 (2005), suppl., $1-31$.

[S07] Siu, Y.-T. Techniques for the analytic proof of the finite generation of the canonical ring. Current Developments in Mathematics 2007 (2009), 177-219. https://projecteuclid.org/euclid.cdm/1254748606

[S09] Siu, Y.-T. Dynamic multiplier ideal sheaves and the construction of rational curves in fano manifolds. In: Complex Analysis and Digital Geometry, Proceedings from the Kiselmanfest, 2006, ed. Mikael Passare. 323-360, Acta Univ. Upsaliensis Skr. Uppsala Univ. C Organ. Hist., 86, Uppsala Universitet, Uppsala, 2009. http://nrs.harvard.edu/urn-3:HUL. InstRepos: 9367003

[S10] Siu, Y.-T. Effective termination of Kohn's algorithm for subelliptic multipliers. Pure Appl. Math. Q. 6 (2010), no. 4, Special Issue: In honor of Joseph J. Kohn. Part 2, 1169-1241. https://arxiv.org/abs/0706.4113

[S17] Siu, Y.-T. New procedure to generate multipliers in complex Neumann problem and effective Kohn algorithm. Sci. China Math. 60 (2017), no. 6, 1101-1128. https://arxiv.org/abs/1703.06257

[W08] Wlodarczyk. J. Resolution of singularities of analytic spaces, Proceedings of 15th Gokova GeometryTopology Conference pp. 31-63. http://gokovagt.org/proceedings/2008/ggt08-wlodarczyk.pdf

[Z19] Zaitsev, D. A geometric approach to Catlin's boundary systems. Annales de l'Institut Fourier, Volume 69 (2019) no. 6, p. 2635-2679. https://doi.org/10.5802/aif.3304

S.-Y. Kim: Center for Mathematical Challenges, Korea Institute for Advanced Study, 85 Hoegiro, Dongdaemun-gu Seoul, Korea

E-mail address: sykim8787@kias.re.kr

D. Zaitsev: School of Mathematics, Trinity College Dublin, Dublin 2, Ireland

E-mail address: zaitsev@maths.tcd.ie 